\title{
An Insight into Information, Entanglement and Time
}

\author{
Paul L. Borrill \\ paul@borrill.com
}

\section{Introduction}

"Church's thesis and the Turing machine are rooted in the concept of doing one thing at a time. But we do not really know what doing is - or time - without a complete picture of quantum mechanics and the relationship between the still mysterious wave-function and macroscopic observation."

- Andrew Hodges in: Alan Turing: Life and Legacy of a Great Thinker [1]

We combine elements of Boltzmann's statistical account of thermodynamic processes in the second law, Shannon's theory of communication, and a background-free conceptualization of time, where the arrival and departure of information carried by photons defines an ordering of events which are perpetually evolving and reversible (therefore perpetually re-ordering) inside isolated entangled systems. This becomes progressively irreversible as decoherence ebbs and flows with the environment.

Our argument brings a new information-theoretic quality to the nature of an interaction. We use this concept in the context of a perpetual symmetric exchange of information between atoms by a photon, where the direction is (at the microscopic level) predictable, yet observation remains non-deterministic because we cannot know (in an individual measurement) how many times a reversal takes place without disturbing the system. The absurd idea is that reality is timeless inside entangled systems, i.e., it continually evolves and cycles through its recurrence, defined by the available number of states. This symmetry can however be broken at the macroscopic level by an observer preparing the system for measurement, triggering causality to select a direction for information and energy to flow.

We introduce subtime $\left(t_{s}\right)$ as a reversible information interchange within an entangled system and reexamine the conclusion dismissed by Einstein, Podolsky \& Rosen (EPR) 2. We accept the principles of relativity and the constancy of the speed of light $c$ (in $t_{s}$ ), but question our ability to measure $c$ with experiments that presume a classical $\left(T_{c}\right)$ smooth, monotonic and irreversible background in time [3.

We offer an alternative view in the spirit of Boltzmann indistinguishability: in addition to the indiscernability of individual particles with identical properties [4] we recognize that states previously visited within a quantum system are indistinguishable from reversing subtime to that prior state.

\section{Information, Photons and Time}

We begin by assuming information is associated with the propagation of a photon ${ }^{1}$ and postulate subtime is inextricably intertwined with space along the one-dimensional path defined by the photon traversal between emitter and absorber atoms (the Shannon transmitter/receiver channel ${ }^{2}$ ). We see no need for $4 \mathrm{D}$ (Minkowski) spacetime within which light cones are projected (in an empty manifold) to reason about causality, non-locality and the ordering of events. In a nutshell, we dispense entirely with the notion that a background of time exists, along with any sense of future or past, between isolated entangled systems. Instead, reversible computation occurs within an entangled system. Only when the entangled system decoheres into the environment of other entangled systems (through the exchange of photons) does time emerge as progressively irreversible, providing persistent evolution of information at the macroscopic scale. 


\section{The Absurd Idea}

We propose a principle of retroactive non-discernability in the recurrence of information states in entangled systems. Subtimes (helicity eigenvalues) are incremented with photon traversals ${ }^{3}$ from Alice to Bob and decremented on their return path resulting in a net zero change in subtime $\left(t_{s}\right)$ while $\left(T_{c}\right)$ stands still $^{4}$.

Instead of the assumed traversal of a photon through the apparatus once only from the source to detector, imagine a photon traversing backward and forward perpetually through the apparatus an arbitrary (uncountable) number of times before it is finally absorbed by an atom in the detector and passed on. We would be unable to detect (in any single measurement) how many traversals actually occurred before we registered the event in $T_{c}$. This implies:

- Most experimental observations would provide no clue that we were not measuring intervals in $t_{s}$. Instead in $T_{c}$ we experience observation events like a quantum stroboscope, which illuminates reality only in quick flashes with long periods of darkness in between.

- Unlimited evolution can take place within an entangled system in subtime, but (a) we would be unable to discern one recurrence from another from our $T_{c}$ vantage point and (b) even for large systems of particles many intermediate configuration states could be visited in its environment and then be reversed to a predecessor state before some external observation registered the state in $T_{c}$.

- Many different configurations are explored in subtime and only those well suited to their environment would (with higher probability) persist as (what would appear to be) irreversible change in $T_{c}$.

\section{Entanglement and Recurrence}

- Two atoms exchanging a photon with each other in perpetuity comprises an entangled system (Figure 1). Each arrival of the photon (in $t_{s}$ ) at the atoms represents a gain in information and departure represents a loss, i.e., entropy. Subtime $\left(t_{s}\right)$ is incremented along the photon's path from the receiver's point of view, and decremented from the point of view of the transmitter.

- Each entangled system can evolve through its configuration space an arbitrary (and uncountable) number of times, but is inevitably constrained to a recurrence which is temporally indiscernible from any previous or successive recurrence.

- When we go from one to two atoms mediated by a photon, subtime $\left(t_{s}\right)$ is an isolated temporal experience of that two party system. As we add more atoms to the system, the number of discernible configurations increases non-linearly. The recurrence of the system becomes richer and more diverse, but the configuration space is still limited by the number of retroactively discernible configurations.

- Every entangled system evolves (and de-evolves) or expands (as it receives new energy) and decays independently (as it loses energy/information to their environment as the system decoheres). We would thus expect some power law distribution. For example, the simplest two atom system would be almost $100 \%$ reversible in its state of perpetuity between recurrences while progressively larger systems of atoms would have both a larger space of recurrences as well as a smaller probability of reversing (or de-evolving) to a previously visited state simply because the number of states is so much larger. The emergence of irreversibility in $T_{c}$ would rapidly approach $100 \%$ as we observe larger and larger objects up the chain to our macroscopic world.
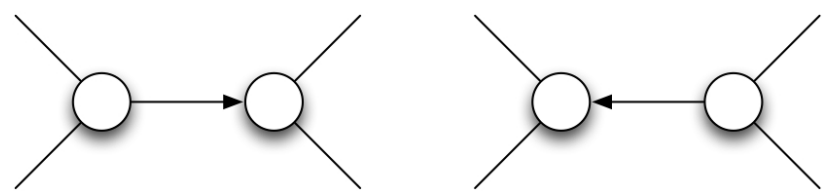

Figure 1: The Heisenberg Cut: Photons Can Go Both Ways (A Hot Potato Protocol In Perpetuity) 


\section{Information and Quantum Mechanics}

Shannon information and Quantum Mechanics (QM) share a common context: probability. And all probability is conditioned on the actions of an observer, i.e., what binary (yes/no) questions the observer asks, either explicitly or implicitly. In QM, the minimum number of states (yes/no answers) needed to fully describe the system is exposed by the experimenter's preparation of the system's apparatus to measure them.

Our initial framework for the insight includes:

1. Information is conserved in the transmission of energy between two entangled entities $A$ and $B^{5}$. This system is perfectly time-symmetric. This includes a causal symmetry for all events (interactions) which we equate to "simultaneous elements of reality" in $\mathrm{EPR}^{6}$. The underlying unitary evolution of the quantum state is computationally reversible.

2. The entangled exchange of information is a perpetual hot potato protocol. It becomes impossible to discern (in any single measurement) the first traversal of information from $A$ to $B$ (or vice versa) from the $N+1^{\text {th }}$ traversal of information, i.e., $\mathrm{N}$ is fundamentally uncountable. This symmetry is broken only when an observation is prepared which triggers the energy and information flow - for example, from side $B$ to $A$ - establishing a casual and thermodynamic direction.

3. Transmitters and receivers are fully symmetric - either party can emit while the other party absorbs ${ }^{7}$. Information transfer is negative with respect to the transmitter and positive with respect to the receiver.

4. There is nothing in nature, or in any measurement carried out so far, which supports the notion of a background of time which would allow us to discern temporal relationships between two or more independent (separable) entangled systems. There is no coordinate system or frame of reference. If a system has no interactions with other systems, there is no common frame of reference in time; total and partial orders are undefined.

\section{Information and Entanglement}

Entanglement of quantum states of composite systems of two or more atoms is a consequence of the principle of superposition. An understanding of this phenomenon has eluded physicists since EPR first drew our attention to its paradoxical nature. Insight to explain the experimental evidence that nature behaves quantum mechanically and non-locally has thus far been elusive.

EPR [2] described two possible explanations to the phenomenon of entanglement: (a) there was some interaction (simultaneous reality) between the particles despite their physical separation or (b) information about all possible outcomes was encoded in hidden variables. EPR preferred the second explanation because the notion of instantaneous action at a distance was in conflict with special relativity.

There is also a third explanation: a flaw in our belief that we can measure time as a smoothly, monotonically increasing point on a continuum ${ }^{8}$.

Time is change. When nothing changes, time stands still, when something changes, and then changes back, it is indistinguishable from time standing still, at least locally.

Entanglement represents a state of reversible change; it is impossible to count the number of exchanges of photons with atoms in the state of entanglement. This is one example of (apparent) randomness in quantum theory. It is not truly random (in the sense of being unpredictable), but it is uncountable because we cannot distinguish a single (one directional) exchange between two entities from any arbitrary odd number of exchanges because they are fundamentally indistinguishable in the measurement events in $T_{c}$.

The orthodox assumptions which may mislead us regarding a global background of time are:

The continuum assumption. The experience of (say) an atom (receiver or transmitter of information) is stroboscopic; information change occurs abruptly at the instant (in $t_{s}$ ) of emission, or absorption of the photon by an atom. 
Although motion may be continuous (down to the Planck limit), it is the arrival of new information that presents a change of state in the receiver. These discontinuous events in $t_{s}$ masquerade as a continuous flow in our assumptions regarding $T_{c}$.

The irreversibility assumption. We implicitly assume from human experience that time marches irreversibly forward. There is no evidence for this in physics. What we do know is that if time (change) happens, we remember. If it happens and then the motion or information reverses its path, we don't remember. Even behaviors that have already decohered in $T_{c}$ which we might think to be immutable once they have happened, can (at least locally) unhappen, within our $T_{c}$ system state, along with our memories being reversed also [5].

An indefinite amount of motion units can be added and subtracted but only the net will be experienced by the observer. Only a witness with perfect single traversal properties would be able to detect the difference in motion units in the system being measured. It is equally likely that the observer is fooled by the same stroboscopic, uncountable but indistinguishable phenomena experienced by the system being measured.

\section{Bell Experiments and Virtual Machines}

Einstein proved that simultaneity was relative, but when we carry out Bell experiments, we set up our apparatus to detect coincidences with a tacit assumption that our observable measurements in $T_{c}$ are equivalent to durations in $t_{s}$. Testing Bell's inequality requires two independent measurements (at points separated in space). Information regarding these measurements is signaled to a common site where coincidence is analyzed [6].

For the purpose of articulating this insight, imagine that virtual machines $\left(\mathrm{VM}^{\prime}{ }^{9}\right)$ are used to carry out the experiments; one each at the separated points and a third at the common site to analyze the signals from the other two for coincidence. These VM's are governed by a clock cycle many orders of magnitude shorter than required to measure and analyze the results (Aspect's atomic clock). In the spirit of Maxwell, imagine a demon ${ }^{10}$, which suspends and resumes each of the virtual machines such that their periods of awareness do not overlap, but their computational state remains available while they are suspended and can be read by the others. The VM's have no independent timing reference, and have no idea that they are being time multiplexed in the $t_{r t}$ (real-time) domain; their entire experience is described by the events they observe in the $T_{v m}$ (virtual machine) domain.

Now further imagine that these VM's are capable of reversible computation, and that the demon can allow the computation to proceed arbitrarily far into the algorithm, but at any time reverse that computation to some prior state visited by that VM. The equivalent of this in the world of computing, is for the VM to be reset to some prior snapshot in order to re-acquire some previously consistent state. The VM has no idea it has been reset. Its only clue might be that its hardware time counters now differ from some external source of time that it may acquire from the network.

We can tune the rate of production of entangled photons such that they occur in the timing window of the measurement VM's, and the statistics of Bell states will emerge. However, this says nothing about what happens outside the timing windows, where any number of internal events my have taken place, i.e., any amount of forward or reverse computational evolution (non-Landauer [7, 8] reversals or resets).

What is actually happening in the real world of Aspect's [9] Bell experiments? The apparent change in correlation (at distance) as soon as the polarizer is switched is explained simply by the reversal of subtime (the photon bouncing back) in $t_{s}$, and a rewriting of history in $T_{c}$, which of course, our instruments and memories would be unable to remember [5] - an example where we can catch nature reversing itself even after we have made an observation. This, implies that relativistic separability remains intact in $t_{s}$, while the temporal artifacts of violations in Bell's inequalities shows up in our $T_{c}$ record $^{11}$.

From this insight, we can now begin to formulate a new and logically consistent information view of the apparent non-locality revealed in violations of Bell's inequality without sacrificing the principle of locality. 


\section{Information and Simultaneity}

Since 1905 we often see assertions that there is no space without time because the constancy of the speed of light provides a limit to velocity of information traversal between points in space. We rarely hear the logically equivalent that there is no time without space which is equally concludeable from Einstein's original postulates and argument [10. Implications of this include:

- The notion of Minkowski space as a 4D manifold can mislead us that time passes, independently of the spatial dimensions. We assert that subtime does not flow when there is no motion in the spatial dimension.

- Simultaneity (Cauchy) surfaces, even in inertial frames, have no basis in reality. There is no common meaning to time separately from motion. They are inextricably tied together.

- Intervals are the measurable elements of space/time, terminated by the atoms on either end of the photon path. Subtime intervals are thus finite. The edges of the subtime graph are summed together to form the emergence of $T_{c}$. This is interesting, because intervals in time have been described by Barbour as an enigma, identified by Einstein as an issue but otherwise remains unresolved [1].

- The only objective reality that can be measured is through interactions - the ultimate locality. Entities must interact (touch, collide, bounce off, be absorbed, emitted etc.) in order to transfer information. However, all the internal interactions of an entangled system are, by definition, unobservable. In $T_{c}$ we observe only those rare events that touch the outside world through decoherence.

- This interaction is fundamentally based on the exchange (gain or loss) of information. For example when an atom receives a photon it gains information, when it emits a photon it loses it (entropy).

- In bipartite entanglements, a photon (and its associated information) is trapped, perpetually bouncing between the atoms (just as it is likely that within an atom, photons are perpetually bouncing between the electrons and protons in the nucleus).

We assume that information is transmitted between particles by photons at a finite speed the maximum being the speed of light, but question our ability to perceive this change as a reversible information-theoretic process. This creates an illusion of superluminal quantum-mechanical processes in experiments designed with a hidden assumption of an absolute time background which hinders our understanding of the EPR paradox.

\section{Definitions}

\section{Subtime $\left(t_{s}\right)$}

Subtime is what happens when we are not looking. It is the perpetual, temporally directionless evolution of information through the interactions of atoms and photons. Subtime is (for our present argument) continuous, and is inseparable from the motion of photons. Everything that happens in subtime can unhappen ( $t_{s}$ is reversible). A photon that travels from Alice to Bob can be followed by a traversal of that same photon from Bob to Alice. The state of the system is now indiscernible from that which existed before the first traversal, or indeed any prior or later traversal of the photon between them.

We describe subtime as propechronus - from the Latin "propinquus"; (of space) near, neighboring, (of time) near, at hand, not far off; and "chronos"; the personification of time. To emphasize its locality and temporally symmetric nature. 


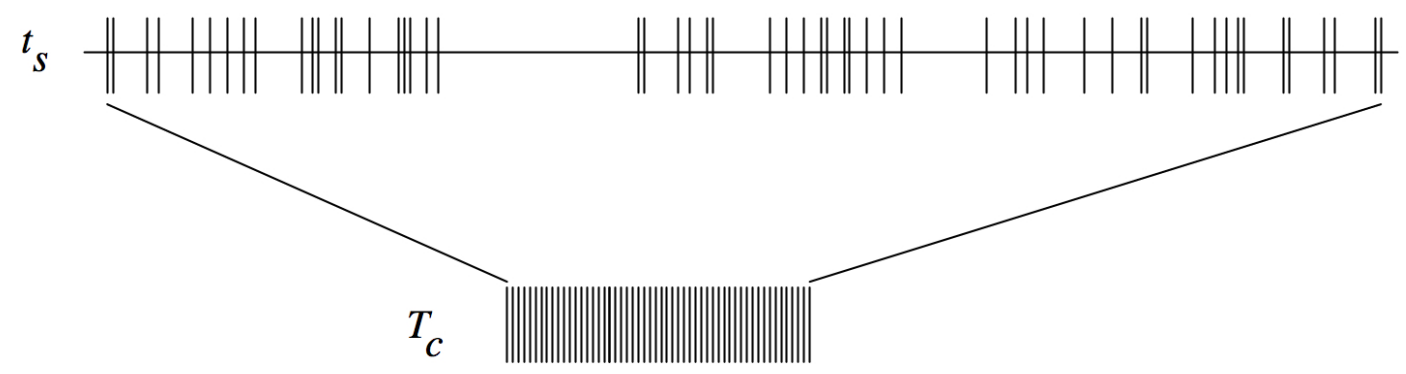

Figure 2: Asynchronous Events in Subtime $\left(t_{s}\right)$ Will Appear Continuous In Classical Time $\left(T_{c}\right)$

\section{Classical Time $\left(T_{c}\right)$}

$T_{c}$ appears successive, monotonic and irreversible and its sign is always positive (because it represents the absolute value of the sum of subtime $t_{c}$ intervals).

The perpetual hot potato photon exchange in entanglement is timeless in $T_{c}$ because we are unable to measure it with our instruments without taking energy out of the system (and thus disturbing the state of entanglement).

Figure 3 below shows a chain of 9 atoms with an information/energy quantum traversing the system. The red path shows one alternative, which accumulates $t_{s 1}$ subtime units, and an alternate (green) path (which continues from half way through the red path before branching in a different direction) accumulates $t_{s 2}$ subtime units. Both will be experienced in $T_{c}$ as the same interval of time.

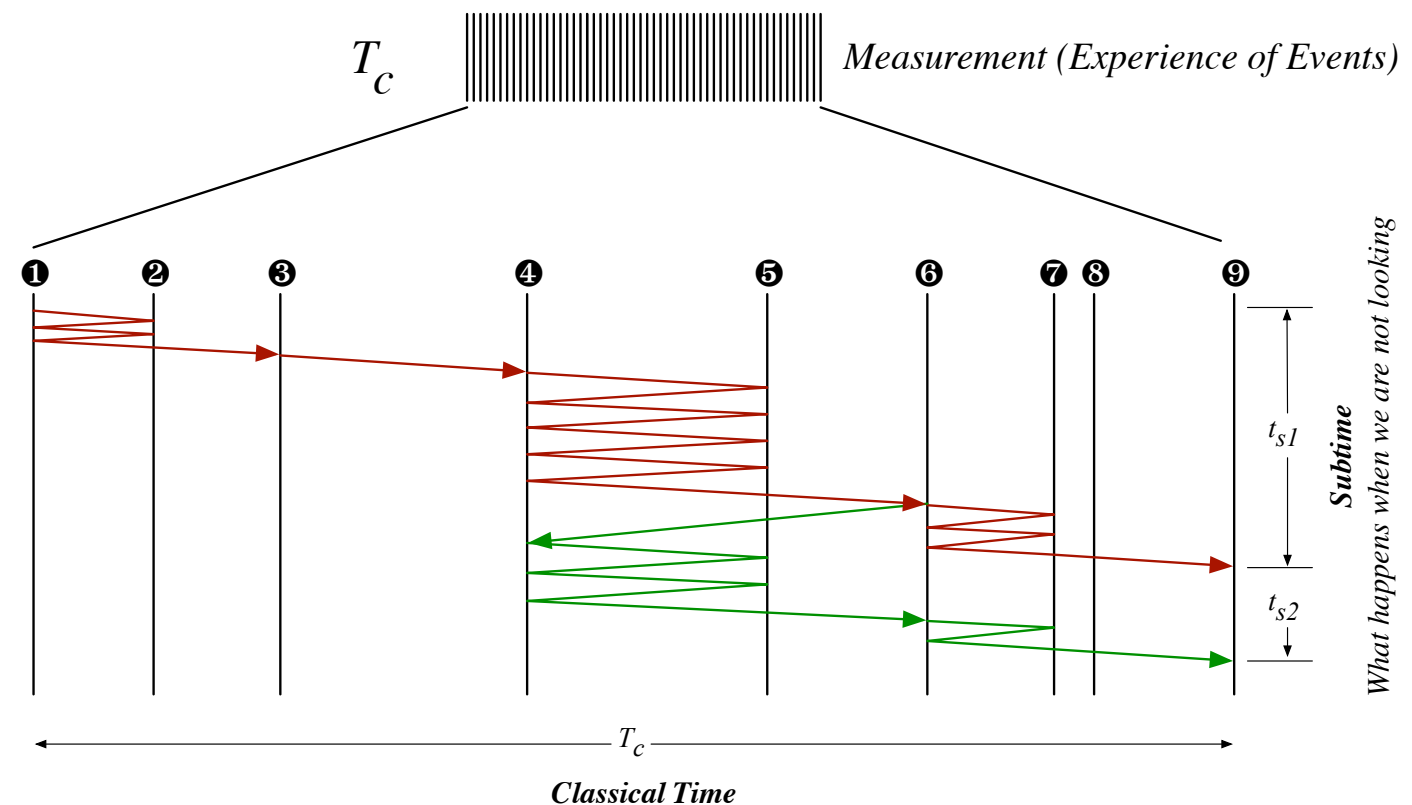

What we, our instruments and computers see

Figure 3: Asynchronous Durations In subtime $\left(t_{s}\right)$ "Can" Appear Shorter In Classical Time $\left(T_{c}\right)$ 


\section{Extending the Entanglement Graph}

Figure 4 below shows a larger system of atoms, with three examples of alternate paths for the information/energy of a photon to travel between the ingress and egress points shown. The total length of the path defines $t_{s}$, but what we observe in $T_{c}$ will be the absolute value of all the increments (forward traversals) of the photon, minus all the decrements (reverse traversals).

There are two segments of path (3) (the 2nd and 4th segments) where we illustrate entanglement as a multiple reflection back and forth. Note that an arbitrary odd number of reflections of this photon is indistinguishable from a single traversal. Also there is no way for us to discern (in a single measurement) whether path (1), (2) or (3) has an arbitrary number of entanglements within them.

- There is no distinction in the passage of time (in $T_{c}$ ) as far as the "outside world" is concerned, with paths (1), (2) or (3).

- Path (3) includes back and forth passing of the information/energy between the vertices in the graph. The number of passings back and forth is uncountable. This mixed path shows both temporary entanglement and direct cut through of photons through the system.

- There is no global passage of time. Each measurement experiences time in $T_{c}$ as the not yet reversed receipt (and passing on) of information/energy.

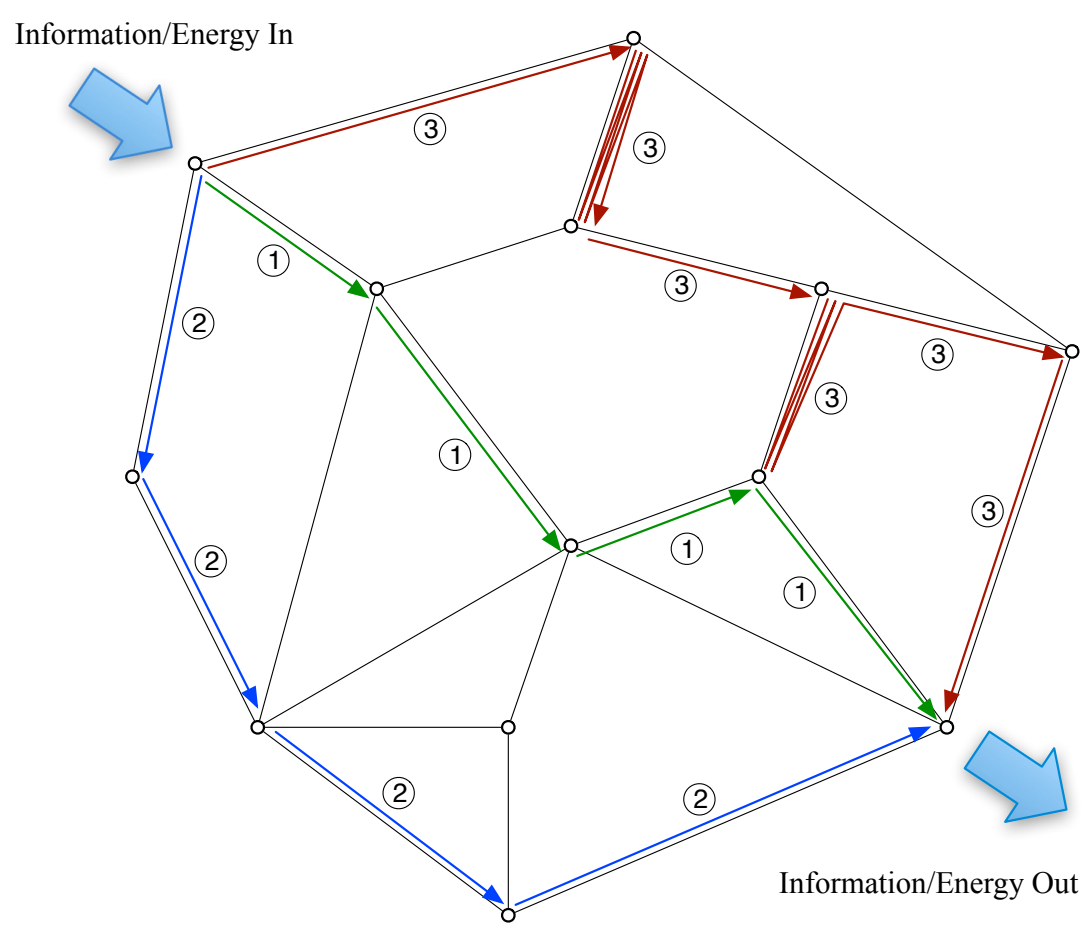

Figure 4: A Graph (2-D) View of Different Paths in An Entangled System

\section{Multiple Slit Experiment}

It is commonly believed that if one decreases the intensity of a beam of light, we will eventually reach the point where only one photon is in transit through the apparatus (say a two-slit experiment) at a time. An implicit but unacknowledged belief that the photon travels only once (one way) through the apparatus from the intended source transmitted to the destination (receiver) whereupon the energy (and information) is captured and the measurement is made. 
We offer an alternative: photons may enter the apparatus and instead of transversing once only in a single direction and being fielded by the detector, it may reflect (or be absorbed/re-emitted) from the detector back to the source whereupon it is reflected again back through the apparatus to the detector. This process may continue an uncountable number of times before finally being extracted from the system as information/energy representing the measurement. The fundamental uncertainty in this process is not purely Heisenberg (although it may masquerade as such) it is the uncountability of the reflections. This uncertainty interferes with our ability to accurately measure the reversed intervals of backtracking photons in $t_{s}$, in any single measurement.

We will use the the excellent description by Deutsch [12 as a canonical orthodox description of the multiple slit experiment. Deutsch makes the classic argument (as did Feynman and many others) that there is only one photon passing through the apparatus "at a time". This implicitly assumes that we (and our experimental apparatus) experience a continuum of progressive (monotonically increasing) time in $T_{c}$.

Deutsch explains this phenomena in terms of the Everett interpretation and invokes a huge number of parallel universes (a multiverse) to explain the interference without a Bohr (Copenhagen) style collapse of the wave function. Within the context of subtime, we can see an element of truth in this intuition. Instead of Deutsch's "huge number of parallel universes, each one similar in composition to the tangible one" we can now imagine a huger number of multithreaded explorations of its $t_{s}$ environment between each observation event in $T_{c}$, which persists objects and events that we and our instruments can directly observe.

Deutsch enumerates the possible number of universes. The largest area that we could conveniently illuminate with a laser might be about one square meter and the smallest manageable size hole size might be a thousandth of a millimeter; so there are approximately $\frac{1}{\left(10^{-6}\right)^{2}}=10^{-12}$ possible hole locations on the screen. $10^{12}$ possible alternative configurations can thus be explored in this system. It is critical to acknowledge that while this may be an approximate number for parallel universes, or with subtime exploring each location of the one square meter once, the entanglement in subtime expressed by our new hypothesis is by its very nature uncountable.

The implications of this include a massive unrealized concurrency under the hood of entangled information/time which is reminiscent of the hoped for parallel computation capacity of quantum computing.

We present a critical change in perspective: Instead of some magic parallel universes being explored which is somehow beyond the relativistic physics of spatiality separated entities in some Bell-type inequality, we can now see ourselves and our instruments as observing the universe through a time lens much like that of a stroboscope or cinematographic projector. Each frame of the film represents a snapshot in time (in $t_{s}$ ) and we can be fooled by our measurements into believing that there is zero time between one frame to the next because all the change appears to occur at once.

\section{Entangled Systems are Dark}

Entangled systems exist for an indefinite number of recurrences where the system (say one atom is the source and another the detector) neither gains nor loses energy/information. These are the states which appear maximally entangled. Their existence will be dark i.e., outside of time - unobservable in $T_{c}$.

In $t_{s}$ the photon may take any and all paths that exist in the apparatus an uncountable number of times between each detection event. Between detection events one photon can keep many atoms busy (e.g. preventing them from absorbing other photons of the same wavelength) thus perpetuating the state of darkness which appears as destructive interference.

As the angle between the photon path(s) through the apparatus departs from the nominal $90^{\circ}$ we will observe Franhofer diffraction through a single slit, and interference through multiple slits. The geometry and mathematics of this is well known but the mechanism traditionally used to explain it (wave interference) may now be compared with the obvious explanations within the subtime context. It is not that a single photon (or other quantum particle) is passing through both slits at the same time it is passing back and forth with an indefinite number of traversals each reversing the effect in $t_{s}$. Appearing to traverse the apparatus only once in $T_{c}$ because of our inability to accurately perceive intervals in $t_{s}$ between detection events in $T_{c}$ that appear to reinforce our smooth, monotonic and irreversible assumptions regarding $T_{c}$. 


\section{Falsifiability}

Many experiments can be conceived to prove this hypothesis incorrect. Below are a few of the unique aspects of this insight that may be tested experimentally:

- Separate (non-interacting) entangled systems will develop (evolve their state within the constraints of recurrence) completely independently. No background of time exists which is common to all systems in the universe.

Experiment \#1 Independent atomic clocks will exhibit random (unexplained) perturbations relative to each other. These jumps will be affected by an increase in electromagnetic coupling to other systems (the onset of decoherence).

- Space and time are inextricably intertwined in the motion of a photon traveling between physical endpoints. The phase of the photon helicity with respect to space is locked into the traversal of the photon itself. When those two endpoints (the atoms) enter into their state of entanglement they will then experience events (information arrival and departure), but will not experience duration (the intervals between events).

Experiment \#2 We can expose the distinction in $t_{s}$ and $T_{c}$ by observing that certain observed events in $T_{c}$ are integer multiples of the space/time traversal within the geometry of the apparatus. What would we see if we varied the distance between the source and detector in units of wavelength of the quantum particle in a Bell experiment?

\section{Conclusions}

The photon is the carrier of time, and the Universe is a network automaton ${ }^{12}$ : a graph of evolving relationships where the vertices represent atoms and the edges represent the hot-potato protocol of a continuously (in perpetuity) bouncing back and forth of a photon. In combination with the concept of subtime, this perspective carries all the hallmarks of entanglement.

The photon entanglements represent intervals of time. Indeed, the only realistic intervals that can exist are those that span the space/time path of the photon and are terminated by the atoms. Intervals in subtime are therefore finite and bounded by the (mostly) symmetric emitter and absorber atoms [13.

What goes on inside entangled systems is both timeless and unobservable. Only interactions (observations) with the outside define the order of events that we see. Entangled systems are Dark.

We turn upside down the idea that massive concurrency exists in quantum computation, and suggest instead, that we have been sampling subtime like a stroboscope: brief flashes of reality with long periods of darkness in between. We also recognize the intuition behind multiple parallel universes, which could instead be an unbounded exploration of the quantum state space, not dissimilar to a conventional computer "multithreading" the many tasks vying for its physical resources and our apparent random selection of the current state of one of the threads through a deliberate (or otherwise) preparation of our observations.

"We must, therefore, be prepared to find that further advance into this region will require a still more extensive renunciation of features which we are accustomed to demand of the space time mode of description."

- Niels Bohr 


\section{References}

[1] C. Teuscher, ed., Alan Turing: Life and Legacy of a Great Thinker. Springer, 2004.

[2] A. Einstein, B. Podolsky, and N. Rosen, "Can quantum-mechanical description of physical reality be considered complete?," Physical Review, vol. 47, pp. 777-780, May 1935.

[3] L. Smolin, "The case for background independence," arXiv e-print hep-th/0507235, July 2005.

[4] S. French and D. Krause, Identity in Physics: A Historical, Philosophical, and Formal Analysis. New York: Oxford University Press, 2006.

[5] L. Maccone, "Quantum solution to the arrow-of-time dilemma," Physical Review Letters, vol. 103, no. 8, 2009.

[6] A. Peres, "Quantum nonlocality and inseparability," arXiv e-print quant-ph/9609016, Sept. 1996. "New Developments on Fundamental Problems in Quantum Physics" ed. by M. Ferrero and A. van der Merwe (Kluwer, 1997) pp.301-310.

[7] C. H. Bennett and R. Landauer, "The fundamental physical limits of computation," Scientific American, vol. 253, no. 1, pp. 48-56, 1985.

[8] C. H. Bennett, "Notes on landauer's principle, reversible computation and maxwell's demon," physics/0210005, Oct. 2002. Studies in History and Philosophy of Modern Physics vol. 34 pp. 501-510 (2003).

[9] A. Aspect, "Bell's inequality test: more ideal than ever," Nature, vol. 398, no. 6724, pp. 189-189, 1999.

[10] A. Einstein, "On the electrodynamics of moving bodies (zur elektrodynamik bewegter korper)," Annalen der Physik, vol. XVII, pp. 891-921, June 1905.

[11] J. Barbour, "The nature of time," tech. rep., FQXi Community, Mar. 2009.

[12] D. Deutsch, The Fabric of Reality: The Science of Parallel Universes-and Its Implications. New York: Penguin Books, 1998.

[13] J. A. Wheeler and R. P. Feynman, "Interaction with the absorber as the mechanism of radiation," Reviews of Modern Physics, vol. 17, pp. 157-181, Apr. 1945.

[14] A. Shimony, "Aspects of nonlocality in quantum mechanics," in Quantum Mechanics at the Crossroads New Perspectives from History, Philosophy and Physics (J. Evans and A. S. Thorndike, eds.), Berlin: Springer, 2010.

[15] D. Hestenes, "Electron time, mass and zitter," tech. rep., FQXi Community, 2008.

[16] C. E. Shannon, "A mathematical theory of communication," The Bell System Technical Journal, vol. 5, no. 1, p. $55,2001$.

[17] J. P. Torres, G. Molina-Terriza, and L. Torner, "Twisted photons: new classical and quantum applications," vol. 5958, SPIE Publications, Sept. 2005.

[18] G. Molina-Terriza, J. P. Torres, and L. Torner, "Twisted photons," Nature Physics, vol. 3, pp. 305-310, May 2007.

[19] J. P. Torres and L. Torner, Twisted Photons: Applications of Light with Orbital Angular Momentum. Weinheim, Germany: Wiley-VCH, 2011.

[20] A. Afanasev, C. E. Carlson, and A. Mukherjee, "Excitation of an atom by twisted photons," arXiv e-print 1304.0115, Mar. 2013.

[21] A. Peres, "Einstein, podolsky, rosen, and shannon," arXiv e-print quant-ph/0310010, Oct. 2003.

[22] A. Aspect, J. Dalibard, and G. Roger, "Experimental test of bell's inequalities using time- varying analyzers," Physical Review Letters, vol. 49, pp. 1804-1807, Dec. 1982. 


\section{Notes}

${ }^{1}$ Almost all the Bell tests so far have been performed with photons 14. However, this description can also be applied to any quantum particle with a deBroglie wavelength. In which case, information simply travels at the slower rate of traversal of the particle through the apparatus. The helical path description is similar for electrons [15.

${ }^{2}$ Shannon [16] defined the notion of channel capacity in his theory of communication. In the process, he also defined the fundamental notion of a "bit" as the fundamental unit of information.

${ }^{3}$ Photons (at least) may be able to transfer multiple bits in higher order angular momentum [17, 18, 19, 20]

${ }^{4}$ This is consistent with the advanced and retarded wave solutions to Maxwell's equations.

${ }^{5}$ We also assume a finite degree in the vertices of a quantum cellular automata graph (each particle represents a vertex) implying:

- A limit to the number of entanglement neighbors for each particle, which can form decoherence partners with the environment.

- This degree limitation, like the valency in atomic bonding, implies that nature builds a multi-hop entanglement network out into the decoherence environment (similar to those in figures $3 \& 4$ ).

- Different particles may have different degrees. For example, photons have degree (2). There can be only one transmitter and one receiver, as in Shannon's channel. Electrons may be likewise, but a spin of $+/-1 / 2$ implies an information configuration space of 2 down its direction of motion, not a degree of 2 , for "elements of simultaneous reality", or the number of entanglements that it may accommodate concurrently.

${ }^{6}$ by "simultaneous" we mean, as assumed in EPR [2, that there must be a coincidence in space, as well as in time, i.e. an interaction.

${ }^{7}$ Subtime $t_{s}$ exists only in the context of space and in only one dimension along the path from transmitter to the receiver (the Poynting vector).

${ }^{8}$ This recognition that the logic of the EPR paper was correct but the assumptions were wrong is shared by Rosen[21].

${ }^{9}$ Virtual machines in computing are software systems that emulate the hardware environment of a real computer, to allow one or many virtual machines (Operating Systems as well as applications) to run on the same physical hardware.

${ }^{10}$ Aspect 22] measured time using randomly switched optical crystals at 50Mhz (20ns), while the spatial extent of the apparatus required more than double that to violate special relativity. This is not what we are referring to as a demon.

${ }^{11}$ There is a insufficient room in this paper to discuss how this hypothesis differs from other "time loophole" theories. We draw the reader's attention to the principal arguments: that subtime starts and stops with the emission and absorption of a photon, and is immediately reversed in all ontological respects as the photon is returned in the hot-potato protocol. This is one way that we divorce ourselves from the background assumption of time, which is not (as far as we can tell) the case for prior time loophole theories.

${ }^{12} \mathrm{~A}$ network automaton is similar to a cellular automaton, but where the cells are vertcies in an arbitrary network, and there is a (possibly evolving) topology of links connecting them. 